\title{
Optical properties of the Ti surface structured by femtosecond laser beam
}

\author{
L. Dolgov ${ }^{1}$, R. Kravchuk ${ }^{2}$, A. Rybak ${ }^{2}$, V. Kiisk ${ }^{1}$, I. Sildos ${ }^{1}$, I. Blonskyi ${ }^{2}$ \\ ${ }^{1}$ Institute of Physics, University of Tartu, 51014 Tartu, Estonia \\ ${ }^{2}$ Institute of Physics, NAS of Ukraine, 46, prospect Nauky, 03028 Kyiv, Ukraine
}

\begin{abstract}
Wavelength-scaled periodic ripples formed on the Ti surface under the action of femtosecond laser irradiation have been investigated. The ripples were oriented in parallel to the incident light polarization. After initial formation of ripples, the following laser induced chemical transformation of metallic Ti into dielectric compound was established using the Raman scattering data. Weak Wood's anomaly in the spectra on light diffracted by the structured Ti surface testifies the minor plasmonic activity.
\end{abstract}

Keywords: laser structuring, titanium, plasmonic waves, Raman spectroscopy.

Manuscript received 28.03.11; accepted for publication 14.09.11; published online 21.09.11.

\section{Introduction}

Formation of periodic structures on metal and semiconductor surfaces by powerful laser pulses is now under the rapt attention. Applied interest is due to the possibility of obtaining periodic surface nanostructures without special mechanical or lithographical performance. Laser induced periodic surface structuring can be used in preparation of diffractive elements [1], active surfaces for enhanced Raman scattering [2], incandescent light sources with improved brightness [3]. According to existing concepts, surface structuring is a result of interrelation between optical, thermal and dynamic electronic processes occurring on the interface during laser irradiation [4-7]. Engaging of different branches of materials science and desire to obtain nanostructures with suitable properties stimulate scientific interest to these laser-structured surfaces.

The most typical laser-induced surface structures are ripples on a sample surface. Physical mechanisms providing formation of ripples may differ in details, therefore they are still staying under discussion. The character of ripples suggests their launching as a result of interference. It may be interference between the incident light and light scattered from surface inhomogeneities [4, 5, 8, 9]. Interference of incident light with induced surface plasmon-polariton waves is also possible $[6,7,10]$. Thus, initial surface roughness possessing the amplitude comparable with the light wavelength is necessary in both cases. Polishing the surface can be used to create initial surface inhomogeneities. In case of plasmon-polariton wave generation, the synchronism condition has to be satisfied: the phase velocity of plasmon-polariton wave induced on the interface must be equal to the phase velocity of incident light. Since surface roughness is changed during laser irradiation, it needs feedback phenomenon providing the development of the relief profile. Such feedback is provided by the temperature difference in the maxima and minima of the interference pattern during the formation of grating. This temperature distribution initiates processes responsible for deepening the relief profile: deformation of surface, melting and evaporation of surface material, possibly, capillary waves on the molten surface or surface acoustic waves $[6,7,10]$. Sometimes, formation of several overlapping relief structures occurs [5-7, 10-12]. In this case, the number of induced surface waves corresponds to the quantity of periodic structures.

Several authors note that the direction of surface ripples depends on the polarization of incident light. Initially, Fröhlich and Sack [13] established the condition later confirmed by other authors [14] that $p$ polarized light stronger interacts with electron plasma in the sample than s-polarized light. In general, the laser induced ripples are transverse to the polarization (RTP) of incident light [4-7, 10-12]. It was recently shown that femtosecond laser pulses allow obtaining several new types of structures with combined nano- and microstructuring [1, 3, 15-19]. In particular, ripples along polarization (RAP) of incident laser light were obtained on the titanium surface $[1,18,19]$. 
This paper is a continuation of our previous work [19] where the conditions for obtaining different laserinduced ripples on the Ti surface were ascertained. Here we investigate the properties and structure of laserinduced ripples in order to evaluate perspectives of using the laser-treated $\mathrm{Ti}$ samples as plasmonically active surface.

\section{Materials and methods}

The femtosecond laser complex based on Ti:sapphire laser generator Mira Optima $900-\mathrm{F}$ was used for irradiation of the sample. Irradiation was performed using the laser beam with the wave-length $800 \mathrm{~nm}$. The duration of each pulse was 130 fs and the repetition rate was $1000 \mathrm{~Hz}$. The energy of one pulse was $0.95 \mathrm{~mJ}$ and the fluence was about $3 \times 10^{11} \mathrm{~W} / \mathrm{cm}^{2}$. Initially, a polished titanium plate made of mark vacuum titanium VT $-1-0$ was used as a target. Polishing was made by diamond paste with the characteristic size of particles $0.5-1 \mu \mathrm{m}$. The residues of polishing material were removed by different solvents: acetone and ethanol. The target prepared in this manner was oriented normally to the laser beam, placed after the focusing lens at the distance $2.25 \mathrm{~cm}$ from the focus and irradiated for $1 \mathrm{~min}$.
The irradiated area of the sample was investigated using optical and scanning electron microscopy as well as optical spectroscopy. Raman scattering spectra from the treated area were recorded employing Renishaw inVia Raman microspectrometer using a blue laser $(488 \mathrm{~nm})$ for excitation. The same system was employed for recording spectra of light diffracted on the surface relief. In this case, the incident beam of white light was obtained by collimating fiber-coupled halogen lamp emission. The light scattered or diffracted by the sample could be detected with spatial resolution down to a few microns owing to the confocal microscopy system.

\section{Results and discussion}

Laser irradiation of $\mathrm{Ti}$ sample induces pronounced ripples in its surface structure. The trace from a laser beam is a dark elliptically shaped area with approximate size $500 \times 900 \mu \mathrm{m}$. At higher magnification, the ripples appear to be composed of a big amount of small unidirectional dark lines with the length about $1 \mu \mathrm{m}$ and thickness less than $1 \mu \mathrm{m}$ (Fig. 1).

These small lines appear near the scratches and roughened areas that left after initial polishing of the sample surface. It is known that initial chaotic surface

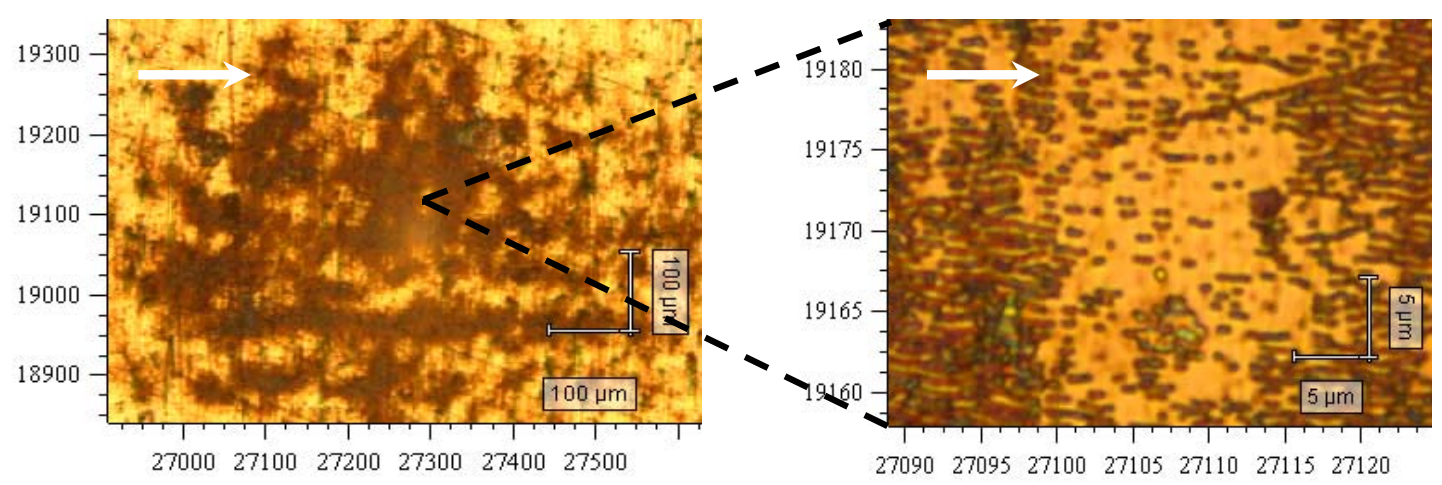

Fig. 1. Optical microscopic images of the laser-treated Ti area at different magnifications. Laser induced lines of relief are parallel to the polarization of laser beam (marked by arrows).

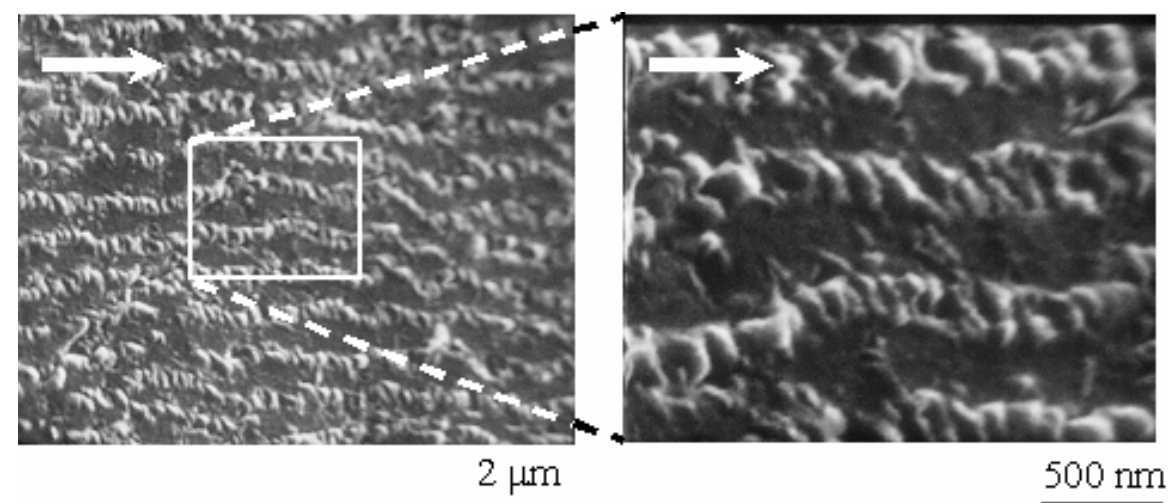

Fig. 2. Scanning electron micrographs of laser treated $\mathrm{Ti}$ area at different magnifications. Laser induced lines of relief are parallel to the polarization of laser beam (marked by arrows). 


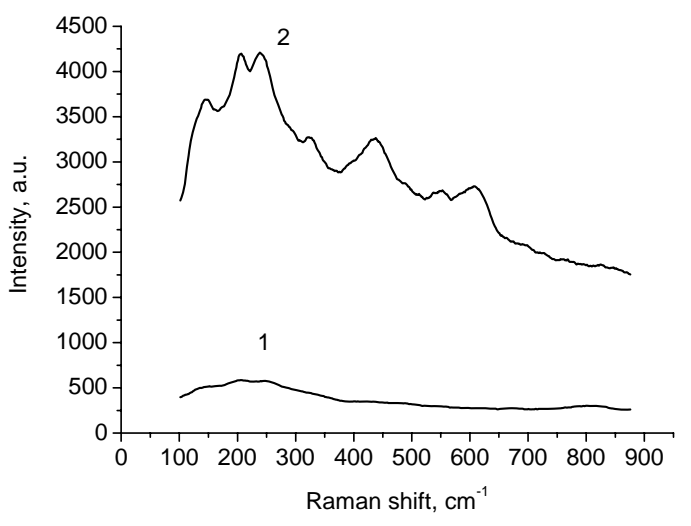

Fig. 3. Raman spectra of light scattered from the different points of laser treated Ti area: (1) away from the relief lines induced by laser; (2) near the relief lines induced by laser.

irregularities commensurable with the thickness of metal skin layer provide conditions for surface plasmonic wave interference with the incident light [4-7]. Scanning electron micrography of laser treated area revealed periodic nano-relief structures with the period $0.8-1 \mu \mathrm{m}$ comparable with the wavelength of incident laser light (Fig. 2).

In contrast to typical structures with ripples transverse to the polarization (RTP) of the incident light, in our case ripples along polarization (RAP) appear like to $[1,18,19]$. Some authors called this type of laser induced relief as "anomalous" [10] or "extraordinary" $[6,11]$, because of its allegedly atypical orientation. Previously, RAP were obtained on the dielectric or semiconductor surfaces such as Ge [5, 8] and glass [11]. In the case of Ti samples, the appearance of RAP is always accompanied by transformation of surface from metal to dielectric state $[1,18]$. It gives us an initial idea that dielectric permittivity may have critical influence on the type of gratings formed on the Ti surface. To unclear the type of transformations induced on Ti surface, we analyzed the Raman spectra of the laser treated area.

Gauss profile of the laser beam intensity results in a higher density of ripples near the center and smaller at the periphery of the irradiated area (Fig. 1). Raman spectra from both areas were recorded with micrometerscale spatial resolution (Fig. 3). Raman scattering from materials with metallic properties is restricted by prohibitive selection rules and a low penetration depth of the incident light. Therefore, Raman bands are very weak in the spectrum obtained from less treated metallic areas of Ti sample (Fig. 3, curve 1). Stronger treated areas demonstrate Raman bands with shifts to the regions $100-300 \mathrm{~cm}^{-1}$ and $400-600 \mathrm{~cm}^{-1}$. These bands can be associated with non-stoichiometric titanium nitride with admixture of oxygen atoms $[20,21]$. Usually, $\mathrm{TiN}_{\mathrm{x}} \mathrm{O}_{\mathrm{y}}$ compound is formed on the surface when Ti interacts chemically with air. The integrated intensity of bands obtained from the points with nano-relief surface is on average 15 times higher than for less structured areas (Fig. 3). Micromapping the Raman signals confirms correlation between pronounced nano-structuring and enhancement in the integrated intensity of Raman peaks (Fig. 4). It seems reasonable that laser treatment stimulates reactions of $\mathrm{Ti}$ with nitrogen and oxygen from air. Thus, there is a bigger amount of dielectric $\mathrm{TiO}_{\mathrm{x}} \mathrm{N}_{\mathrm{y}}$ compounds in the treated areas.

In their previous papers, Akhmanov et al. [6] and Emel'yanov et al. [10] proposed the plasmonic theory for RAP formation, which is based on interrelation between laser-induced interference and evaporation from the surface of the sample. They pointed that formation of laser-induced RAP occurs in materials with the real part of dielectric permittivity situated within the region $-1<\varepsilon<1$. These values of $\varepsilon$ can be obtained in the plasma escaped from the metal surface under action of
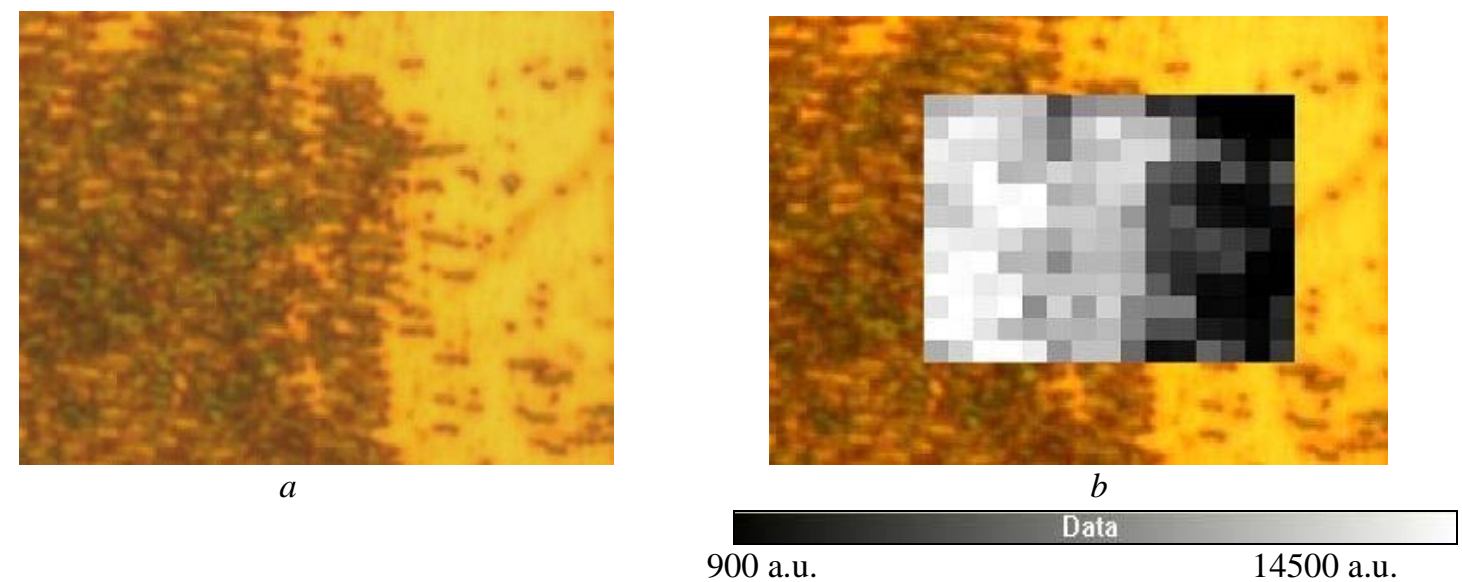

Fig. 4. Microscopic image of laser treated Ti area: (a) in reflected white light; (b) the same area of the sample with overlapped mapping of the integrated signal from Raman bands. The gray level scale indicates the relative intensity. 
femtosecond laser pulses [22]. It is this jump in dielectric permittivity from metallic to the dielectric type that can provoke formation of RAP and the following chemical transformation of surface from metallic to dielectric compound. There is also another point of view considering the special types of surface defects and laser induced polarization and light scattering on the surface as main reasons for RAP formation $[4,5,8,9]$. Thus, the general model explaining formation of laser induced RAP is under discussion.

Considering that the interaction of laser irradiation with surface plasmon waves can give rise to the surface structuring, one is naturally curious whether surface plasmons could be optically excited on this structured surface. The answer to this question would be important from the practical standpoint (preparation of SERSactive surfaces, etc.) and also allow us indirectly estimate the degree of surface transformation. Induction of surface plasmons is possible in the case of light diffraction on the periodical metal surface. In this case, spectra of diffracted light have a dip at a plasmonic resonance wavelength $\lambda$, because of partial transformation of light energy into the surface electromagnetic wave. This dip in the spectra is known as a Wood's anomaly [23, 24]. Therefore, the specific plasmon dips in the spectra of light diffracted on lasertreated surface can reveal the unreacted part of Ti. It is possible to estimate which angle of incidence $\theta$ can result in inducing the plasmonic wave, if knowing the dependence of real part for $\mathrm{Ti}$ permittivity $\varepsilon$ on the photon energy [25] and the synchronism condition relation [26]:

$n_{D} \sin \theta+m \frac{\lambda}{d}= \pm \sqrt{\frac{\varepsilon \cdot n_{D}^{2}}{\varepsilon+n_{D}^{2}}}$,

where $n_{D}$ is the refractive index of the environment over the grating, $m$ is the order of diffraction, $d$ is the period of grating. Registration of the diffracted light partially transformed into the plasmon waves can be realized at angles $\varphi$ satisfying the equation [26]:

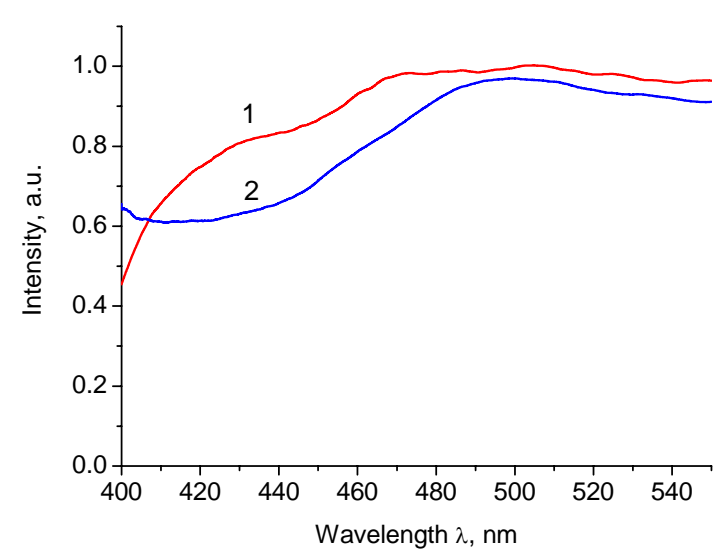

Fig. 5. Relative intensities of light diffracted at the angle $\varphi$. The angle of incident $p$-polarized light is $\theta$ : (1) $\theta \approx 40.5^{\circ}$, $\varphi \approx 3^{\circ},(2) \theta \approx 44.5^{\circ}, \varphi \approx 7^{\circ}$. $m \frac{\lambda}{d}=n_{D}(\sin \theta-\sin \varphi)$.

For example, equations (1) and (2) predict excitation of a surface plasmonic wave for $\mathrm{Ti}$ grating with $d=0.8 \mu \mathrm{m}$ by the first order diffraction $(m=1)$ at $\theta \approx 40^{\circ} 37^{\prime}, \quad \varphi \approx 3^{\circ} 8^{\prime}, \quad \lambda \approx 477 \mathrm{~nm}$ and corresponding $\varepsilon \approx-2.8$. In this case, we observed diffraction of a polarized and collimated beam of broadband light. The experiment was done in two geometries where the plane of incidence was either perpendicular or parallel to the surface relief lines. Initially $p$-polarized light was especially sensitive to plasmonic effects in the considered geometries. Relative intensity of diffracted light $(m=1)$ in case of initial $p$-polarization is presented for experimental cases $\theta \approx 40.5^{\circ}, \varphi \approx 3^{\circ}$ (Fig. 5, curve 1 ) and $\theta \approx 44.5^{\circ}, \varphi \approx 7^{\circ} \quad$ (Fig. 5, curve 2). The plasmonic minimum in the intensity is predicted at $\lambda \approx 459 \mathrm{~nm}$ in the latter case. One can see that the intensity of $p$-polarized light decreases in the expected plasmonic region $459 . . .477 \mathrm{~nm}$. But plasmonic decrease in the intensity is not very sharp and pronounced in comparison with reflection dips obtained for traditionally used noble metals $[24,26]$. This pure plasmonic response can be explained by the fact that our grating is non-regular on the macroscale (Fig. 1), and most of the surface area is transformed to a dielectric state.

\section{Conclusions}

The ripples on $\mathrm{Ti}$ surface were produced by the femtosecond laser irradiation. They consist of the regular, on the micro-scale, lines directed parallel to the polarization of incident light. Results of Raman measurements suggest that laser treatment induces not only ripples on the surface, but also following transformation of metallic $\mathrm{Ti}$ into dielectric $\mathrm{TiO}_{\mathrm{x}} \mathrm{N}_{\mathrm{y}}$ compounds. Partial chemical transformation limits usability of the laser treated $\mathrm{Ti}$ as plasmonically active surface. Therefore, laser treatment of samples in the inert atmospheres can be considered as possible improvement in future investigations.

\section{Acknowledgements}

We greatly acknowledge Center for collective use of "Laser femtosecond complex" associated with Institute of Physics of NASU for the hardware support. We thank kindly V. Styopkin from the department of Physical electronics, Institute of Physics NASU for the SEM investigations of the samples. This work was supported by European Social Fund grant GLOFY0102J and Estonian Science Foundation grants numbered as 7456 and 6999.

\section{References}

1. S. Camacho-Lopez, R. Evans, L. Escobar-Alarcon, M.A. Camacho-Lopez, Polarization-dependent 
single-beam laser-induced grating-like effects on titanium films // Appl. Surf. Sci. 255, p. 3028-3032 (2008).

2. E.D. Diebold, N.H. Mack, S.K. Doorn, E. Mazur, Femtosecond laser-nanostructured substrates for surface-enhanced Raman scattering // Langmuir, 25, p. 1790-1794 (2009).

3. A.Y. Vorobyev, V.S. Makin, C. Guo, Brighter light sources from black metal: significant increase in emission efficiency of incandescent light sources // Phys. Rev. Lett. 102, 234301 (2009).

4. J.E. Sipe, J.F. Young, J.S. Preston, H.M. van Driel, Laser-induced periodic surface structure. I. Theory // Phys. Rev. B, 27(2), p. 1141-1154 (1983).

5. J.F. Young, J.S. Preston, H.M. Driel, J.E. Sipe, Laser-induced periodic surface structure. II. Experiments on Ge, Si, Al, and brass // Phys. Rev. B, 27(2), p. 1155-1172 (1983).

6. S.A. Akhmanov, V.I. Emel'yanov, N.I. Koroteev, V.N. Seminogov // Sov. Phys. Uspekhi, 28, p. 1084 (1985), doi: 10.1070/PU1985v028n12ABEH003986

7. A.M. Bonch-Bruevich, M.N. Libenson, V.S. Makin, V.V. Trubaev // Opt. Eng. 31(4), p. 718-730 (1992), doi: 10.1117/12.56133

8. J.F. Young, J.E. Sipe, J.S. Preston, H.M. Driel // Appl. Phys. Lett. 41(3), p. 261-264 (1982).

9. P.A. Temple, M.J. Soileau // IEEE J. Quantum Electr. QE-17(10), p. 2067-2072 (1981).

10. V.I. Emel'yanov, E.M. Zemskov, V.N. Seminogov // Sov. J. Quantum Elect. 14(11), p. 1515-1521 (1984),

doi:10.1070/QE1984v014n11ABEH006482

11. F. Keilmann, Y.H. Bai // Appl. Phys. A, 29, p. 9-18 (1982).
12. Y. Huang, S. Liu, W. Li, Y. Liu, W. Yang // Opt. Express, 17(23), p. 20756-20761 (2009).

13. H. Fröhlich, R.A. Sack // Proc. Phys. Soc. 59(1), p. 30-33 (1947), doi:10.1088/0959-5309/59/1/305

14. M. Maillard, P. Monchicourt, M.P. Pileni // Chem. Lett. 380(5-6), p. 704-709 (2003).

15. V. Oliveira, S. Ausset, R. Vilar // Appl. Surf. Sci. 255(17), p. 7556-7560 (2009).

16. A.Y. Vorobyev, C. Guo // Appl. Phys. Lett. 92, 041914 (2008).

17. A. Vorobyev, C. Guo // Phys. Rev. B, 72, 195422 (2005).

18. B. Oktem, H. Kalaycioglu, F.Ö. Ilday // Proc. of CLEO, San Jose, CA, May 16, 2010, http://www.opticsinfobase.org/abstract.cfm?URI=C LEO-2010-CMR4

19. A. Rybak, R. Kravchuk, V. Styopkin, I. Pavlov, I. Blonskii // Book of abstracts NANSYS 2010, p. 613, ISBN 978-966-02-5783-2.

20. A. Trenczek-Zajac, M. Radecka, K. Zakrzewska, A. Brudnik, E. Kusior, S. Bourgeois, C.M.de Lucas, L. Imhoff // J. Power Sources, 194, p. 93103 (2009).

21. W. Spengler, R. Kaiser // Solid State Communs. 17, p. 19-22 (1975).

22. Yang Yang, Jianjun Yang, Lu Xue and Yan Guo // Appl. Phys. Lett. 97, 141101 (2010).

23. R.W. Wood // Phys. Rev. 48, p. 928-937 (1935).

24. H. Raether, Surface Plasmons on Smooth and Rough Surfaces and on Gratings. Springer-Verlag, Berlin, Heidelberg, New York, London, Paris, Tokio, 1988.

25. D.W. Lynch, C.G. Olson, J.H. Weaver // Phys. Rev. $B, 11(10)$, p. 3617-3624 (1975).

26. O. Telezhnikova, J. Homola // Opt. Lett. 31(22), p. 3339-3341 (2006). 